\title{
Development of Mobile-Based Intelligent Surgery Information System
}

\author{
Shen-Yuan Tang \\ Department of Information System \\ Shin Kong Wu Ho-Su Memorial Hospital \\ Taipei, Taiwan \\ Department of Computer Science and Information \\ Engineering \\ Tamkang University \\ Taipei, Taiwan \\ e-mail: A000896@ms.skh.org.tw
}

\author{
Chien-Liang Chen \\ Department of Computer Science and Information \\ Engineering \\ Aletheia University \\ Taipei, Taiwan \\ e-mail: clchen@au.edu.tw \\ Lun-Ping Hung \\ Department of Information Management \\ National Taipei University of Nursing and Health \\ Sciences \\ Taipei, Taiwan \\ e-mail: lunping@ntunhs.edu.tw
}

\begin{abstract}
Nowadays, medical industry actively develops mobilebased information system to improve service quality. It can not only reduce writing errors caused by human negligence, but also reduce nurse's and physician's workload and elevate the quality of patient care. The study was conducted at a medical center located in northern Taiwan. Through the establishment of the proposed mobile-based information system, documents used in surgeries are eliminated and are recorded in computers. This mobile-based information system combines HIS, PACS and LIS to assist medical personnel to import patient's clinical records, imaging reports, inspection reports and other relevant information before, during and after surgery. We propose the record, re-check, report and reference, the $4 \mathrm{R}$ checking mechanism as the core concept of the development of the proposed mobile-based intelligent surgery information system. The checking mechanism can automatically view the completion of medical record, and maintain a high degree of integrity if the records are incomplete. After launching the system one and half year, we conduct a questionnaire survey and collect data from nurses and physicians who use this system. The survey was designed to measure the successfulness of the mobile-based information system. Questions focused on three broad areas: information quality, system quality, and users' satisfaction. The results confirmed a high degree of satisfaction among users. According to the feedback, it shows that the system should improve the function of automatic saving on regular basis.
\end{abstract}

Keywords-mobile-based surgery information system; operating room; questionnaire survey

\section{INTRODUCTION}

When performing a complicated surgery, health care personnel's use of the computer input method not only ensures the efficient completion of various records needed to complete the surgery, but also enhances the completeness and accuracy of the data input. However, occurrences of medical negligence, such as wrong operation sites, patients whose identities have not been checked, incorrect surgical methods and processes, and other risks [4] continue to take place. As surgery is an important part of medical treatment, from the time a patient is wheeled into the operating room, every check and confirmation done by the nursing personnel has an impact on the patient's safety. Hence, improving surgical procedures is considered an important safety indicator in enhancing surgeries of patients [1] The traditional surgical support system merely provides a simple interface design without providing users good operating results on using the system. Human errors and loopholes in system checking mechanisms can easily affect the accuracy and completeness of follow-up medical records. Some papers proposed the development of a surgical system that involves the use of a new type of information technology to assist health care personnel to handle various records needed during the surgery. In addition, in terms of performance validation of the information system, numerous thesis papers adopted the questionnaire analysis to evaluate the users' degree of satisfaction and the effectiveness of operation [3]. Moreover, in terms of measuring the successfulness of the system, DeLone and McLean [2] proposed six dimensions for evaluating the successfulness of the information system. They are system quality, information quality, service quality, user satisfaction, and use and net quality. Because using the proposed system is mandatory in clinical practice, the hospital set up a policy requiring operating room related health care personnel to use this system when making records. In our work, the service quality, use and net quality did not have direct impacts on this study. Only the system quality, information quality, and user satisfaction were adopted in this work as the assessment variables.

In this work, we attempt to establish a mobile-based integrated system to enhance patients' safety when seeking medical attention and to facilitate the transparency of the 
hospital's information. Based on 4R systematic checking mechanism, it is an integrated computer-aided surgery report system. Through the questionnaire distribution method, the health care personnel's satisfaction for system use was studied to gain an insight into improving the data record completeness and suggestions for other applications, thereby effectively eliminating the insufficiencies and strengthening the feasibility of the mobile-based surgical information system.

\section{SYSTEM ENVIRONMENT}

General surgical procedures are divided into three stages: preoperative, intraoperative, and postoperative stage as show in figure 1 . On the day of the surgery, control personnel of the operating room notify the nursing stations to check the room number and patient's identity, to complete all preoperative processes, and to wheel the patient into the operating room. In order to ensure patient safety, the scrub nurse and mobile nurse should first confirm data with the patient before the anesthesiologist administers anesthetics. Before the surgery, doctors, anesthetic nurse, scrub nurse, and mobile nurse will first suspend their work on hand to engage in "time out" action. This step is the most important preoperative aspect: confirm patient identity, surgical method, surgical site, and related medical staff list. After the surgery, the patient is to be wheeled into the recovery room to wait for the anesthesia to subside. Then, the respective nursing stations shall commence postoperative care.
In this work, the mobile-based integrated computer-aided surgery reporting system developed based on the 4R mechanism was put forward. The proposed surgical information system is a web-based interface in mobile device, divided into three modules: preoperative, intraoperative, and postoperative modules. Before an operation, the nursing personnel can view the surgery schedule record for the day. During the operation, this system can be adopted to input the surgical patient's physiological information, the number of surgical appliances used, surgery-related information, and nursing records. Physician can write the operation records. During the operation, the system can be connected to the Picture Archiving and Communication System (PACS), Laboratory Information System (LIS), Radiological Information System (RIS), and Hospital Information System (HIS) to access information of the surgical patient. The surgical patient's postoperative data can also be accessed by the outpatient clinics and nursing stations, thus facilitating the tally and pricing of back-end supplies, as well as for educational purposes such as case teaching and research. It not only clearly and conveniently keeps written records of the surgery-related data, but also provides confirmation and reminder mechanisms to ensure the completeness of the items that need to be properly filled out. Meanwhile, the reconfirmation function also ensures the subsequent attribution of responsibility and the effective retention of the knowledge and experiences throughout the surgical procedures, thereby facilitating future research development. The information system design processes are as shown in figure 1.

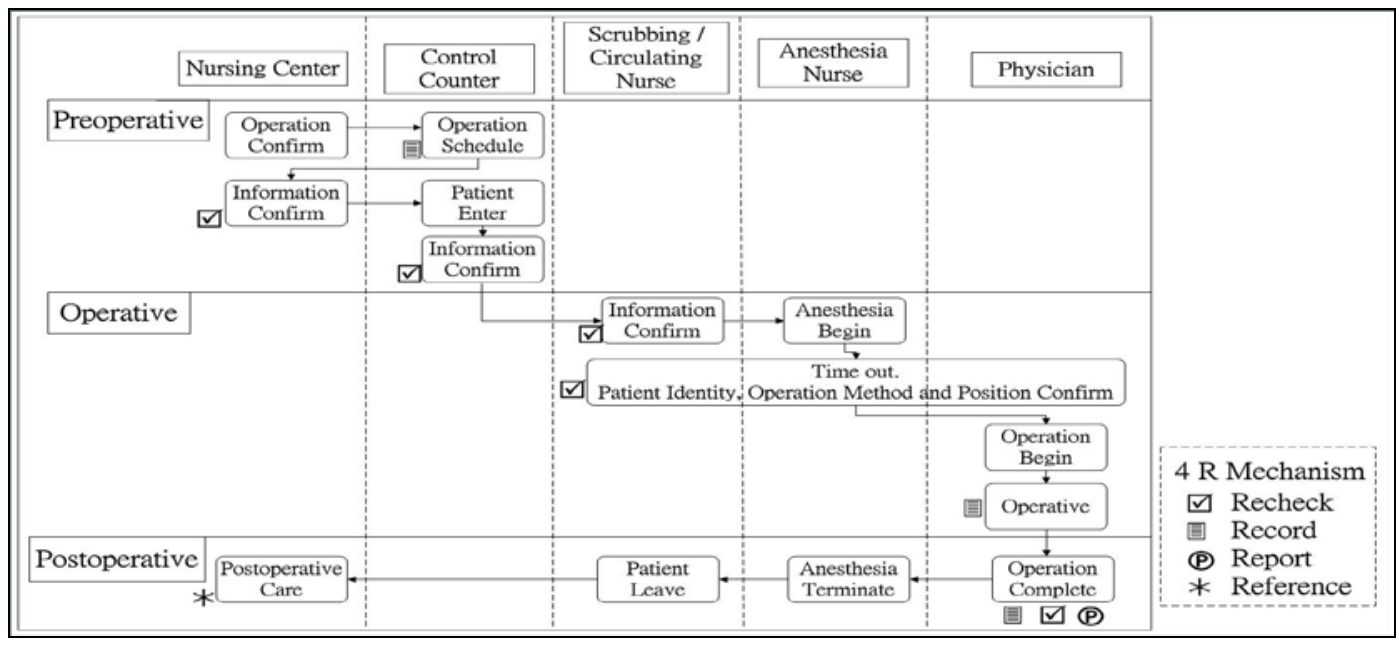

Figure 1. The process of surgical information system.

\section{THE CONSTRUCTION OF THE INTERGRATED COMPUTER-AIDED SURGERY}

The 4Rs as the core functions were put forward, including: Recording, Rechecking, Reporting, and Reference. Important steps of the surgery were included in the development of objectives that integrates the patient's surgery schedule, detailed records of the surgery, records of the surgical care, surgical record sheet and report retrieval system and ensure the effective use of the people, things, and objects in the surgical procedures.

\section{A. Recording Function}

As shown in figure 2, in this system, the record data was divided into surgery schedule, surgery detail records, surgical care records, and surgical record sheet. The surgery records must be completed as soon as a surgery is completed, while the 
unified format allows the user to write on the same table and enables the viewer access to neat and uniformed report.

The surgical schedule has a list of the patients to undergo surgery on the day, which saves the nurse's time in checking. The screening function also helps find the data needed. In the surgery detail records, the patients' basic information can be found, the names of the physicians and nurses involved in the surgery are recorded. In this frame, the patient's operating room number, medical record number, and name are indicated. This allows the nurse to record the time by pressing the "time button" when performing an action. There are five checkpoints: the time the patient arrives at the waiting area in front of the surgery room, the time the patient enters the operating room, the time the patient is administered anesthesia, the time the surgery is ready to begin, and the time of the preoperative "Time Out."

The surgical care record covers four sections, namely, marking the surgical site and special object placement site, checking data, appliance logon, object confirmation, and surgical care detail records. The main purpose of marking the surgical site and special object placement site is to doublecheck the surgical marks on the patient before the surgery, so as to prevent medical disputes due to operating on the wrong site. Regarding the appliance logon, the number of appliances used during the surgery is recorded. The usage of the appliances may be recorded many times during the surgery. At the end of the surgery, the system will automatically add the appliances used for the nurse to conveniently tally and confirm, at the end of which the checker's name is inputted and the personnel in charge of the appliance counting is recorded for the purpose of responsibility attribution. In addition, the patient's anesthesia method, drugs and antibiotics administered, the surgery posture taken by the patient, and other nursing records are recorded to accurately keep track of the content of every item during the surgery.

Finally, the surgery record sheet aids in medical treatment and serves clinically as a diagnostic reference. Hence, the system framework mainly consists of text and images. The system especially features the requirement that the physician has to complete the current patient records before being allowed to move on to the next surgery for the next patient. As for physicians, need, they may use the sample reference function to fill out the page, through which the system automatically retrieves the diagnostic contents of similar surgeries for the physician to make quick corrections and deletions. The system's mandatory mechanism has changed physicians' past habit of writing. As for patients, it serves as a protection, as it prevents record data from being tampered with. After inputting the data, the writer's personal account and password have to be supplied, and this record sheet does not allow modifications.

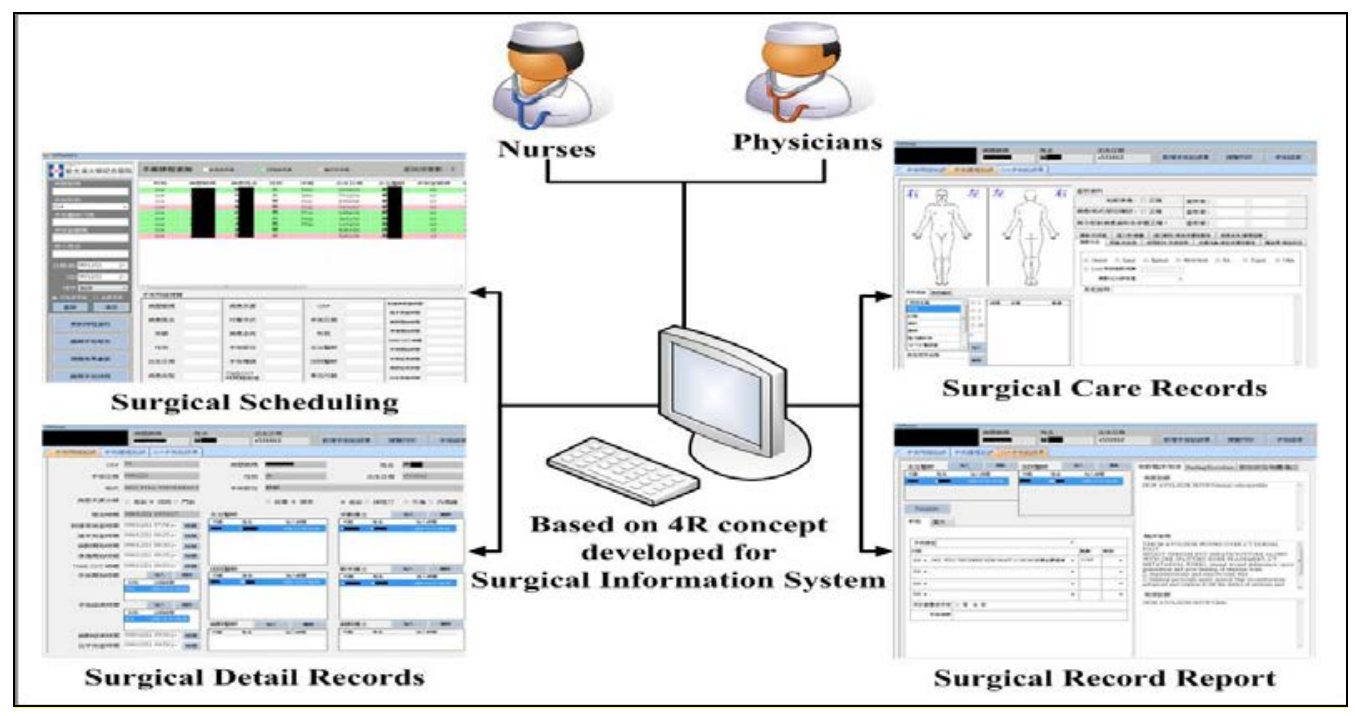

Figure 2. The recording function.

\section{B. Rechecking Function}

The surgical procedures are divided into three re-checking time points, and the checking is performed targeting the time, appliance used, surgical site, and Time Out. The three time points are: before the induction of anesthesia, before the surgeon makes the first incision, and before sending the patient out of the operating room. At these three important time points, some important information needs to be reconfirmed, including: before the induction of anesthesia, the nurse should verbally ask for the patient's name and surgery site at the surgery waiting room, make sure that the surgery and anesthesia consent forms are completed, and ensure the safety of the surgery and anesthesia administration. Before the surgery commences, the nurse should check the anesthesia equipment, drug assessment, and surgical site again, which is considered an important part of the preparation. As shown in the singlelined red box in figure 3 , the nurse should check the items in the data column and affix the signature. This stage ensures the 
smooth conduction of subsequent steps and is thus extremely important and should not be ignored.

After the patient enters the operating room, the physician must cease all actions before making the first incision and implement the "Time Out" procedure. The physician should confirm the patient's name, surgical apparatuses, instruments, instrument tray, the patient's X-ray, and other preparatory work. The surgeon should verbally ask the surgical team members, so as to ensure the patient and correctness of the related data are properly confirmed by all personnel involved. After entering the physicians' and nurses' names and codes and confirming the Time Out procedure, then the scrub nurse can hand over the surgical knife to the surgeon and start the operation. Finally, before sending the patient out of the operating room after the surgery, the counting action is conducted three times in the operating room. The first counting is conducted before the surgery to obtain the inventory of the various apparatuses on the instrument tray, and the second counting is conducted when all the supplies on the instrument tray during the surgery have been used up and additional quantities are needed. The system

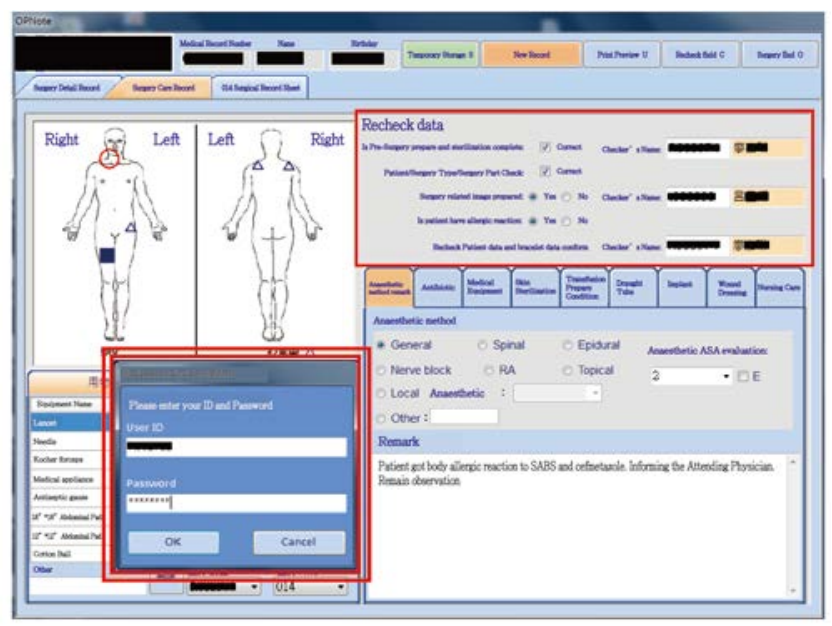

Figure 3. The surgical care records.

\section{Reporting Function}

The detailed medical history contents filled out before, during and after the surgery should be confirmed for errors and omissions by a nurse to facilitate the automatic acquisition of information needed. By clicking "print", three forms: the surgery record (OP NOTE) as the reference for determining prices, the surgery record sheet (OP RECORD) as the doctor's advice, and the surgery care record sheet as a file saved by nurses for future reference. As shown in figure 4, after clicking the single-lined red box "signature", the double-lined red box frame in figure 4 will appear. After entering the nurse's employee I.D. number and password, confirming and clicking "OK" indicate the report has completed the electronic signature. That is, subsequent modifications will not be allowed, and only browsing can be executed. To health care personnel, this is an undeniable proof. As to the patient, the health care personnel's alteration of the doctor's advice and nurse's advice leading to medical disputes can be prevented. will insert the time an additional record entry is made. Finally, at the end of the surgery, the system will calculate the total number of tools used during the surgery. As shown in the double-lined red box in figure 3, after the nurse took inventory, the pop-up window containing the serial number and code entered appeared for confirmation. Compared to the conventional way of tallying the usage using five strokes, nurses today can check and count whether the inventory matches the actual quantity, so as to make sure no apparatuses are accidentally left inside the patient. After wheeling the patient out of the operating room, surgeons and nurses must engage in shift matters, including reconfirming the patient's name, the name of the surgery, the blood pressure changes before and after administering anesthesia, the surgery situation, the blood loss, the wound condition, etc. They must also advise on matters to note during the recovery and treatment of the patient. After the entire surgery is completed, the system will automatically perform a check to detect omissions the nurse has failed to fill out. In case of omissions, the system will send out a warning to ensure the completeness of the system contents.

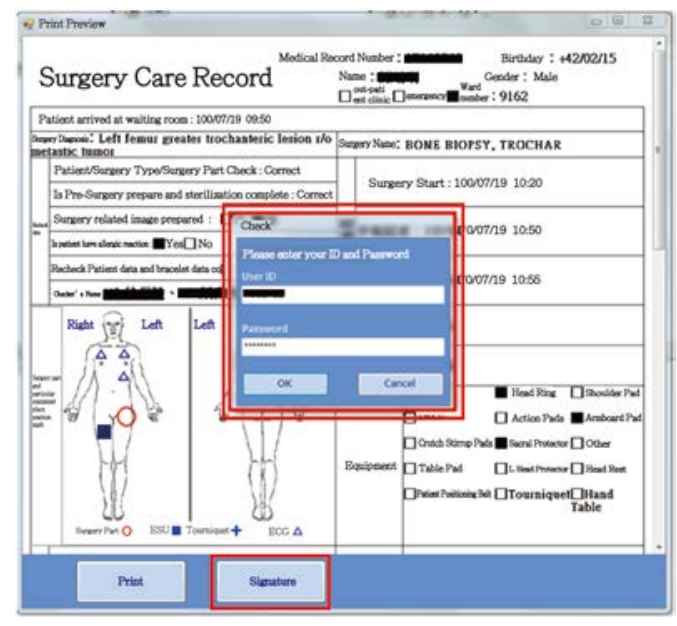

Figure 4. The surgical care records report.

\section{Reference Function}

All the medical records of patients shall serve as research references provided without security concerns. In addition, through the related medical records, important information such as the current medical situation or epidemic trend can be understood. In a complete surgical process, many images and report records can be made available to future intern doctors for learning and other reference purposes, such as updates and breakthroughs in surgical methods, whether or not the patient's days of hospitalization are reduced, and whether there is a significant reduction in complications compared to past years, or which departments have higher clinic rates based on the individual observations of the department to determine the epidemic trend of a particular year, and other statistics can be completely analyzed through the surgery information system. As shown in figure 5, the patient was diagnosed with urethral stricture by the physician before the surgery, but after the surgery the disease was found to be cystitis. Hence, this 
medical history possessed a high referential value and shall be provided to future intern physicians for more in-depth clinical medical research. In terms of the reference function of the clinically provided image records and pathological and anatomical diagnosis, Specimen Picture Import provided the surgeons with relevant handling pictures and pathological tissue collections during surgeries. Through the touch screen, the sites of the collections and surgical site contents were sketched. As shown in figure 6, the surgery specimen images were photographed. For example, the photograph of the lipoma specimens were converted into the Digital Imaging and

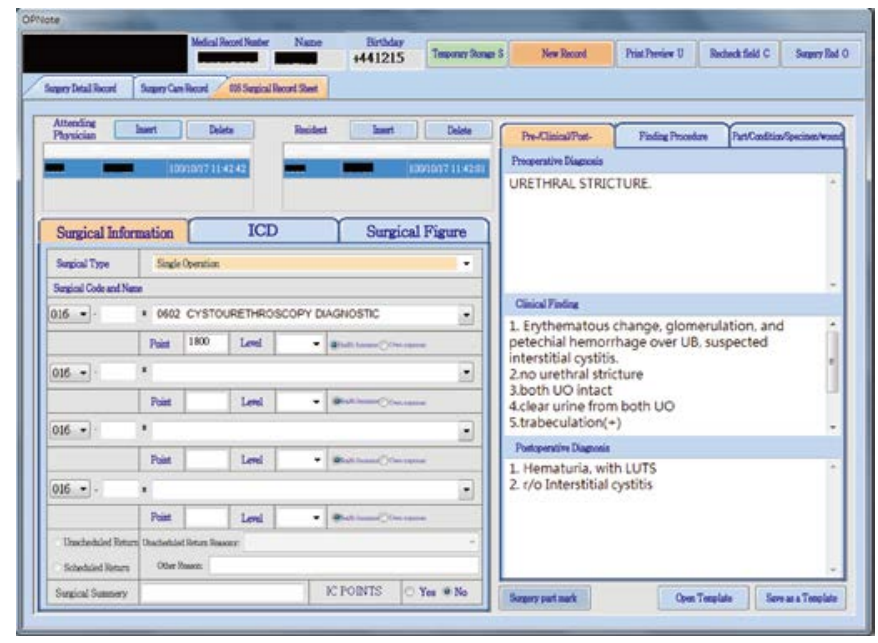

Figure 5. Reference - surgical record sheet.

\section{THE QUESTIONNAIRE DESIGN AND DISCUSSION}

To understand the feasibility of the surgery information system established for health care personnel and feedbacks such as the degree of satisfaction, a questionnaire survey was conducted.

This questionnaire targeted a teaching medical center in northern Taiwan, and the subjects were physicians and nurses that adopted the surgery information system. The questionnaire contents are divided into three parts. The first part is the collection of the demographic data collected from the health care personnel. The second part is the degree of satisfaction for the surgery information system.

The last part consists of open-ended questions. The design of the open-ended question is intended to find the user's other opinions and ideas when using the system and to explore user's actual needs and difficulties encountered. In the research, a total of 120 questionnaires were distributed, and the valid response rate was $83 \%$. The three main dimensions of the questionnaire are: system quality, information quality, and users, respectively. In the questionnaire of 17 questions, in order to prevent the respondents from being unable to clearly express their degree of satisfaction when filling out the questionnaire, leading to the incorrect application and interpretation of the research results, the four-point Likert scale was adopted, which included: "strongly disagree”, “disagree”,
Communications in Medicine (DICOM) format and uploaded to the hospital's Picture Archiving and Communication Systems (PACS). The physicians in the pathological examination division then had access to the surgeons' relevant information in pictures and text through the Laboratory Information System (LIS) which served as a reference for clinical consultation. As for the design of the database system, the specimens and surgery photographs uploaded to the PACS system are filed in patients' account and served as a reference for clinical physicians' academic research, verification, and teaching.

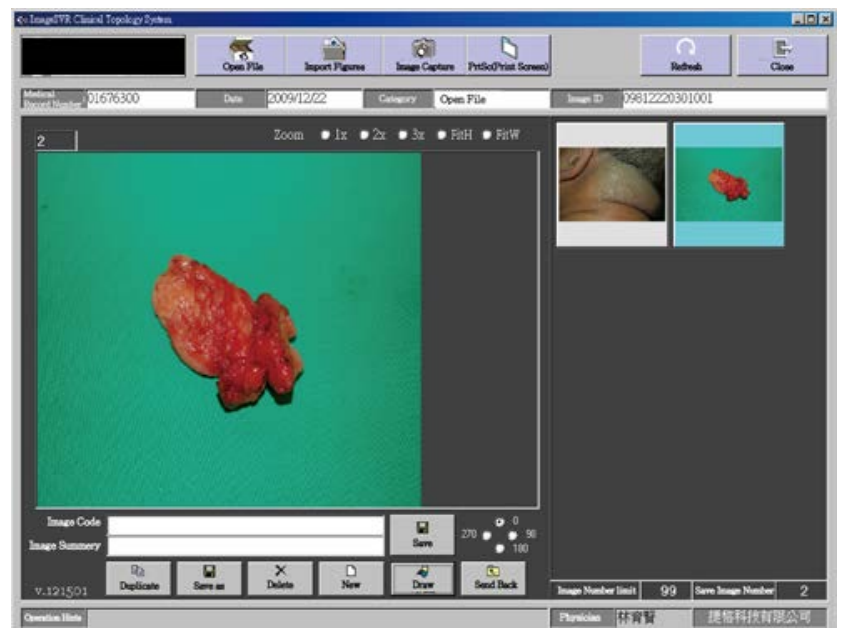

Figure 6. Uploading the photograph of a specimen.

“agree”, and "strongly agree”, with total scores ranging from 17 to 68. A higher score represented a higher degree of satisfaction. Among them, two questions were reverse topics. After the implementation of the proposed system one and a half years later, the overall satisfaction fell in the score range of "satisfied". The item with the highest score was "complete functions provided by the surgery record system", with the average score of 3.03, followed by "satisfied with the overall surgery record system", with a score of 3.00, indicating the health care personnel was highly satisfied with the overall surgery information system and its functions. The reverse question "long response time when the surgery record system is running" had the lowest score. In the open-ended questions, "the inputted data cannot be automatically stored at fixed time interval, and data will be lost if the computer is crashed" accounted for the majority. Concerning the completeness of the nursing records, random samples of 300 copies of medical records before and after the system setup were selected. Before the system setup, the nursing records' mean completeness rate was $88.7 \%$ ( $\mathrm{SD}=15.9)$, while the overall completeness rate of the nursing records was increased to $100 \%(\mathrm{SD}=0)$ after the surgery information system setup. The system's checking mechanism enabled every medical historical copy to be automatically checked after each surgery to determine if the nursing personnel made omissions. Pop-up windows also appear as reminders, which significantly enhanced the completeness rate of the medical records. 


\section{CONCLUSIONS}

An integrated computer-aided surgery reporting system was designed and developed based on the 4R mechanism. This system was implemented and tested clinically for a year and a half. The completeness rate of the medical records increased from $88.7 \%$ to $100 \%$, and the health care personnel that adopted the system to write and retrieve data saves the time of writing forms and reduces the occurrence of errors, thereby enhancing patient safety and health care quality. In addition, this mechanism allows all of the information collected during the surgery recorded and shared perfectly. This system also helped save the printing costs of surgical record sheets and surgical care record sheets, about 100,000 pieces of paper or NTD 63,900 each year.

As far as application is concerned, the surgery information system saves the work time of health care workers, but the inability to automatically store information inputs at fixed time interval should be improved. In order to ensure more diverse functional design of the surgery information system, it is suggested that follow-up researches engage in in-depth discussions toward this direction.

\section{ACKNOWLEDGMENT}

This project received fully support of the Shin Kong $\mathrm{Wu}$ Ho-Su Memorial Hospital to facilitate the completeness of this project. Hereby, we would like to express the highest appreciation from our research team.

\section{REFERENCES}

[1] Chieh-Yu Liu, Ya-Hui Kuo, Mary Etta Mills, Jian-Guo Fong, Cheyu Hung Ting-Ting Lee, "Application of data mining to the identification of critical factors in patient falls using a web-based reporting system," international journal of medical informatics, 2010.

[2] DeLone W. H. and McLean E. R., "The DeLone and McLean Model of Information System Success: A Ten-Year Update," Journal of Management Information Systems, vol. 19, no. 4, pp. 9-30, Spring 2003.

[3] Dexter, F., Epstein, RH., "Operating room efficiency and scheduling, " Curr Opin Anaesthesiol, vol. 18, no. 2, pp.195-198, 2005.

[4] L Lingard, S Espin, S Whyte, G Regehr, G R Baker, R Reznick, J Bohnen, B Orser, D Doran, E Grober, "Communication failures in the operating room: an observational classification of recurrent types and effects," Qual Saf Health Care, vol. 13, no. 5, pp. 330-334, Oct 2004.

[5] Sébastien Grange,Terrence Fong, Charles Baur, "M/ORIS: A MEDICAL/OPERATING ROOM," in ICMI '04 Proceedings of the 6th international conference on Multimodal interfaces, New York, USA, 2004, pp. 159-166.

[6] Sheetal Agarwal, Anupam Joshi, Tim Finin,Yelena Yesha,Tim Ganous, "A Pervasive Computing System for the Operating Room of the Future," vol. 12, pp. 215-228, March 2007.

[7] Ting-Ting Lee, Mary Etta Mills , Barker Bausell, and Ming-Hui Lu , "Two-stage evaluation of the impact of a nursing," International Journal Of Medical Informatics, pp. 698-707, 2008.

[8] Zohar,E.,Noga,Y.,Davidson,E.,Kantor,M.,Fredman,B., "Perioperative patient safety: correct patient, correct surgery, correct side- a multifaceted, cross-organizational, interventional study.," Anesth Analg, vol. 105, no. 2, pp. 443-447, Aug 2007. 